\title{
Ovarian cancer cell invasiveness is associated with discordant exosomal sequestration of Let-7 miRNA and miR-200
}

Miharu Kobayashi ${ }^{1}$, Carlos Salomon ${ }^{1 *}$, Jorge Tapia ${ }^{2}$, Sebastian E Illanes ${ }^{1,2}$, Murray D Mitchell ${ }^{1}$ and Gregory E Rice ${ }^{1}$

\begin{abstract}
Background: The role of exosomes in the pathogenesis and metastatic spread of cancer remains to be fully elucidated. Recent studies support the hypothesis that the release of exosomes from cells modifies local extracellular conditions to promote cell growth and neovascularisation. In addition, exosomes may modify the phenotype of parent and/or target cell. For example, sequestration of signaling mediators into exosomes may reduce their intracellular bioavailability to the parent cell thereby altering cell phenotype and metastatic potential. The fusion of released exosomes with target cell and delivery may also modify cell function and activity. In this study, to further elucidate the role of exosomes in ovarian cancer, the release of exosomes from two ovarian cancer cell lines of different invasive capacity and their miRNA content of exosomes were compared. The hypothesis to be tested was that ovarian cancer cell invasiveness is associated with altered release of exosomes and discordant exosomal sequestration of miRNA.
\end{abstract}

Methods: High (SKOV-3) and low (OVCAR-3) invasive ovarian cancer cell lines were used to characterize their exosome release. SKOV-3 and OVCAR-3 cells were cultured (DMEM, 20\% exosome-free FBS) under an atmosphere of $8 \% \mathrm{O}_{2}$ for 24 hours. Cell-conditioned media were collected and exosomes were isolated by differential and buoyant density centrifugation and characterised by Western blot (CD63 and CD9). Exosomal microRNA (let-7a-f and miR-200a-c) content was established by real-time PCR.

Results: Exosomes were identified with by the presence of typical cup-shaped spherical vesicle and the expression of exosome markers: CD63, CD9. SKOV-3 cells released 2.7-fold more exosomes (1.22 $\pm 0.11 \mu \mathrm{g} / 10^{6}$ cells) compared to OVCAR-3 $\left(0.44 \pm 0.05 \mu \mathrm{g} / 10^{6}\right.$ cells). The let-7 family miRNA transcripts were identified in both ovarian cancer cell lines and their exosomes. The let-7 family transcripts were more abundant in OVCAR-3 cell than SKOV-3 cells. In contrast, let-7 family transcripts were more abundant in exosomes from SKOV-3 than OVCAR-3. miR-200 family transcripts were only identified in OVCAR-3 cells and their exosomes.

Conclusions: The data obtained in this study are consistent with the hypothesis that the releases of exosomes varies significantly between ovarian cancer cell lines and correlates with their invasive potential.

Keywords: Ovarian cancer, Exosomes, microRNA, Biomarkers, Invasion

\footnotetext{
* Correspondence: c.salomongallo@uq.edu.au

${ }^{1}$ Centre for Clinical Diagnostics, Royal Brisbane and Women's Hospital, University of Queensland Centre for Clinical Research, Building 71/918, Herston, Queensland 4029, Australia

Full list of author information is available at the end of the article
} 


\section{Background}

Ovarian cancer continues to be the leading causes of death among women with gynaecological cancer with 225,500 new cases reported each year, causing over 140,200 deaths annually worldwide [1]. Although ovarian cancer accounts for $3 \%$ of all cancer incidents, $6 \%$ of cancer-related death is caused by ovarian cancer, making it the fifth leading cause of cancer mortality in women [2]. While progress has been achieved in our understanding of aetiology and risk factor for ovarian cancer research, there has been little improvement in survival rates [2]. Ovarian cancer continues to be a challenging public health issue.

The keystone to improving health outcomes remains the timely and accurate diagnosis of the predisposition to, or early detection of disease. Early detection of disease risk and onset is the first step in implementing efficacious treatment and improving patient outcome. In the context of ovarian cancer, despite recent progress in chemotherapeutic treatments, the diagnosis of late stage disease is associated with a five-year survival rate of $\sim 30 \%$. In contrast, when ovarian cancer is identified at an early stage, five year survival increases to $\sim 90 \%$ [3]. Thus, the development of more accurate and earlier detection tests for this disease is undoubtedly the number one priority for achieving long- term reduction of mortality from ovarian cancer. To date, such tests have not been developed and the use of traditional biomarker approaches and screening methods have failed to deliver significant clinical outcomes. New approaches to the development of in vitro diagnostics that deliver greater diagnostic sensitivity are required.

Recent studies highlight the putative utility of tissuespecific nanovesicles (e.g. exosomes) in the diagnosis of disease onset and treatment monitoring $[4,5]$. To date, there are only limited data defining changes in the release, role and diagnostic utility of ovarian cancer-derived exosomes.

Exosomes are small $(40-90 \mathrm{~nm})$ membrane vesicles of endocytic origin. The biogenesis of exosomes is initiated by the inward budding of multivescular bodies (MVB), encapsulating cellular proteins and RNA molecules to form internal vesicles. Exosomes are released from several cell types including cancer cells into the peripheral circulation and are detected in biofluids such as plasma, saliva, urine and breast milk [6,7].

Tumour-derived exosomes may affect cancer progression in several ways. Tumour-derived exosomes have the ability to propagate oncogenic activity among tumour cells.

Exosomes are not only mediators of cell-to-cell communication but also represent an attractive source of cancer biomarkers due to the following features: exosomes are (i) actively released from living tumour cells; (ii) convey information about tumour state; (iii) easily obtained from biofluids; easily isolated from high-abundance proteins that confound biomarker discovery; and (iv) are high stability. Most importantly, exosomes are being secreted from living tumour cells and are distinct from apoptotic cell-derived microvesicles [8]. As exosomes contain cellular protein and RNA molecules in cell type-specific manner, they may provide extensive information about the signature of the tumour [9]. Exosomes have been reported to express a diverse range of cell surface receptors, proteins (including, heat shock proteins, cytoskeletal proteins, adhesion molecules, membrane transport and fusion proteins) and miRNA with the potential to affect the acute and long-term function of the cells with which they interact. miRNA is a class of small (approximately $22 \mathrm{nt}$ long), non-coding RNAs that negatively regulate gene expression by binding to the 3' untranslated region of target mRNAs $[10,11]$. Once the miRNA is bound, the target messenger RNA (mRNA) is either cleaved for degradation or its translation is inhibited [12]. miRNAs are evolutionary conserved across species, reinforcing the vast influence of miRNAs on essential biological processes such as differentiation, proliferation, apoptosis [10,12,13]. Deregulation of these miRNAs will not only impact normal physiological processes but also implicated in diseases including cancer. Previous studies have established the significant difference in ovarian cancer miRNA profiles, reinforcing miRNA as a promising cancer biomarker, most studies, however, have examined the miRNA profile of tumour tissues. The collection of tissue samples is an invasive procedure and unsuitable for a diagnostic and screening tests. The utility of cell-free miRNA in biofluids has been investigated as a source of cancer biomarkers. Although this approach overcomes the issue of sample collection, the question remains on how miRNAs are released and avoid degradation. Currently, limited data are available on the mechanism of free miRNA release. The origin of these miRNAs remains unclear and they may be released from apoptotic cells. If this is the case, free miRNAs may not be a useful indicator of tumours state and/or progression.

The let-7 family of miRNAs comprises 10 mature isoforms and is important in development and cell fate control. They are initially expressed as primary (pri)miRNA in the form of a hairpin loop and the base is removed by RNaseII enzyme, Drosha, to form pre-miRNA. PremiRNA is then exported from nucleus to cytoplasm where another RNaseII enzyme, Dicer, cleaves the loop region to produce the mature miRNA. The mature miRNA is incorporated into the RNA induced silencing complex as a guide for target mRNA [14]. Let-7 expression is deregulated in aggressive high-grade ovarian cancer [15]. In addition, miR200 family (i.e. miR-200a, miR-200b and miR-200c) has been associated with ovarian cancer progression [16]. We hypotheses that (i) the release of exosomes from ovarian cancer cell lines is responsive to changes with invasiveness capacity; and (ii) exosomal miRNA content is cell type specific. 
The hypothesis to be tested was that ovarian cancer cell invasiveness is associated with altered release of exosomes and discordant exosomal sequestration of miRNA. Two ovarian cancer cell lines of epithelial cell origin but with different invasive potentials (SKOV-3 and OVCAR-3) were used to test the hypotheses.

\section{Methods}

\section{Ovarian cancer cell lines}

This study was approved by the Human Research Ethics Committees of the Royal Brisbane and Women's Hospital, and the University of Queensland (HREC/09/QRBW/14). All experimental procedures were conducted within an ISO17025 accredited (National Association of Testing Authorities, Australia) research facility. All data were recorded within a $21 \mathrm{CRF}$ part 11 compliant electronic laboratory notebook (Irisnote, Redwood City, CA, USA). The human ovarian cancer cell lines OVCAR-3 and SKOV-3 (with more invasive capacity than OVCAR-3) [17] were obtained from the American Type Cell Collection and were cultured in RPMI media (Life technologies, USA) supplemented with $10 \%$ fetal bovine serum (FBS; PAA, Australia). Cell cultures were incubated at $37^{\circ} \mathrm{C}$ in $8 \% \mathrm{O}_{2}$ and $5 \% \mathrm{CO}_{2}$ atmosphere. Cell confluence and morphology were routinely checked, cells washed with phosphate buffered saline (PBS; Life technologies, USA) and fresh growth media was added every two to three days. Cells were subcultured with dissociation media, TrypLE ${ }^{\mathrm{m}}$ Express (Life technologies, USA) and cellular viability was determined by Trypan Blue exclusion and Countess ${ }^{\circ}$ Automated cell counter (Life Technologies, USA).

\section{Invasion assay of OVCAR-3 and SKOV-3}

Cell invasion rates were established using our previously published method $[18,19]$. Using a real-time cell imaging system (IncuCyte $^{\mathrm{TM}}$ (Essen BioScience, Michigan, USA). In brief, 96-well plates were coated with a thin layer of collagen by transferring $300 \mu \mathrm{g} / \mathrm{ml}$ of collagen type I (Life Technologies ${ }^{\mathrm{TM}}$, Carlsbad, CA) and incubating at $37^{\circ} \mathrm{C}$ for $30 \mathrm{~min}$. OVCAR-3 and SKOV-3 $\left(2 \times 10^{4}\right.$ cells per well $)$ were grown to confluence in complete growth media. Cellfree zones were created by generating a wound with a 96-Well WoundMaker ${ }^{\text {TM }}$ (Essen BioScience, Michigan, USA). The cells were overlaid with $3 \mathrm{mg} / \mathrm{ml}$ collagen type I (Life Technologies $^{\mathrm{Tm}}$, Carlsbad, CA) and incubated at $37^{\circ} \mathrm{C}$ for 30 min to create a 3D matrix. Complete growth media was added on top of the layer of collagen. Cells were imaged automatically every $3 \mathrm{~h}$ over a time period of $48 \mathrm{~h}$. The images were processed by the IncuCyte ${ }^{\mathrm{Tm}}$ software package (Essen BioScience, Michigan, USA) to measure cell invasion by obtaining the Relative Wound Density (RWD, as defined by custom algorithms within the IncuCyte ${ }^{\mathrm{Tm}}$ software package). These users informed algorithms identify the wound region and provide visual representations of the segmentation parameters. Image collection was created using three to five representative phase contrast images.

\section{Exosome isolation from cell-cultured media}

Prior to exosome isolation, cell cultures grown above $70 \%$ confluence were washed with PBS and exosome-free media was added. Exosome-free media composed of RPMI media supplemented with 20\% exosome-depleted FBS and 5\% antibiotic-antimycotic (Life technologies, USA). Exosomes were removed from FBS by ultracentrifuging at 100,000 $\times g$ for $21 \mathrm{~h}$ at $4^{\circ} \mathrm{C}$ and filtering the resulting supernatant with $0.22 \mu \mathrm{m}$ filter (Millipore, Massachusetts, USA). The cells were incubated in exosome-free media for $24 \mathrm{~h}$ at $37^{\circ} \mathrm{C}$ in $8 \% \mathrm{O}_{2}$ and $5 \% \mathrm{CO}_{2}$ atmosphere. The following day, exosomes were isolated from cell-cultured media by differential ultracentrifugation as previously described $[18,19]$ Cell-conditioned cultured media was centrifuged successively at increasing speed: (i) $300 \times g$ for $5 \mathrm{~min} 4{ }^{\circ} \mathrm{C}$, (ii) $1200 \times g$ for $10 \min 4^{\circ} \mathrm{C}$, (iii) $10,000 \times g$ for $30 \min 4^{\circ} \mathrm{C}$, (iv) $100,000 \times g$ for $75 \mathrm{~min} 4^{\circ} \mathrm{C}$. After each step, the pellet was discarded and the supernatant was quantified and used for the following step. The resulting pellet after the fourth step was resuspended in PBS and washed at $100,000 \times g$ for $70 \mathrm{~min} 4^{\circ} \mathrm{C}$ to remove any contaminating proteins. The pellet was resuspended in PBS, layered on a cushion of $30 \%(\mathrm{w} / \mathrm{v})$ sucrose and centrifuged at $110,000 \mathrm{~g}$ for $75 \mathrm{~min}$ for purification. The fraction containing OVCAR-3 and SKOV-3 exosomes ( 3.5 ml, 1.127 density using OPTi digital refractometer (Bellingham ${ }^{+}$ Stanley Inc., Lawrenceville, GA, USA) was recovered with an 18-G needle and diluted in PBS, and then centrifuged at $110000 \times \mathrm{g}$ of $70 \mathrm{~min}$.

\section{Cell count and viability}

Cells were washed with PBS and dissociated with TrypLE ${ }^{\mathrm{m}}$ Express (Life technologies, USA). The number and viability of cells were determined to further calculate the amount of exosome release from one million cells. Collected cells were incubated with Trypan Blue, cell number and viability were assessed using a Countess ${ }^{\bullet}$ automated cell counter (Invitrogen ${ }^{\mathrm{TM}}$, USA).

\section{Exosomal and cellular protein quantification}

Amount of exosomes secreted were estimated by measuring the total protein present within exosomes by the BIO-RAD DC ${ }^{\mathrm{Tm}}$ Protein Assay (USA). Exosome samples were prepared by adding RIPA buffer directly to exosomes suspended in PBS and sonicated at $40^{\circ} \mathrm{C}$ for $15 \mathrm{~s}$ three times to lyse exosome membrane and solubilise the proteins. Bovine serum albumin (BSA) diluted in RIPA and PBS mixture (1:1) were prepared as protein standards at $0,200,400,600,800,1000,1500 \mu \mathrm{g} / \mathrm{mL}$. Standards and samples (prepared exosomes and cell lysate) were transferred to 96-well plates and procedures 
outlined by the manufacture were followed. In brief, alkaline copper tartrate solution (BIO-RAD, USA) and dilute Folin Reagent (BIO-RAD, USA) were added to the samples and incubated for $15 \mathrm{~min}$. The absorbance was read at $750 \mathrm{~nm}$ with Paradigm Detection Platform (Beckman Coulter, USA).

\section{Western Blot}

Exosome markers were identified by Western blot according to our previously published methods [18]. In brief, $10 \mu \mathrm{g}$ of exosomal proteins were separated on NuPAGE 4-12\% Bis-Tris Gel (Life Technologies, USA). Separated proteins were transferred to Immobilon- ${ }^{\circledR} \mathrm{FL}$ polyvinylidene difluoride membrane (Millipore, Billerica, MA, USA) in transfer buffer (5\% NuPAGE ${ }^{\oplus}$ Transfer buffer, 20\% methanol) for 1 hour at $100 \mathrm{~V}$. The membrane was washed in wash buffer (PBS TWEEN 0.1\%) three times for $10 \mathrm{~min}$ and blocked with 5\% skim milk in PBS TWEEN (0.1\%) for an hour at room temperature under agitation. The blocked membrane was further probed for previously identified exosome-specific markers: primary mouse monoclonal anti-CD63 (1:1000, Abcam $\left.{ }^{\circ}\right)$ or anti-CD9 (1:1000, Abcam $\left.{ }^{\oplus}\right)$. This was done by incubating the membrane in primary antibody diluted in 5\% skim milk in PBS TWEEN (0.1\%) at $4^{\circ} \mathrm{C}$ overnight on the laboratory rocker. After an overnight incubation, the membrane was washed with wash buffer and exposed to secondary antibody, donkey anti mouse IgG-HRP (1:1000, Santa Cruz Biotechnology) diluted in $5 \%$ skim milk in PBS TWEEN (0.1\%) for $1 \mathrm{~h}$ at room temperature under agitation. The membrane was washed 3 times for $10 \mathrm{~min}$ in wash buffer. Bound antibodies were detected by enhanced chemiluminescence (peroxide buffer and enhancer buffer, Thermo Scientific) and visualised with the SRX-101A Tabletop Processor (Konica Minolta, Ramsey, NJ, USA).

\section{Transmission electron microscopy}

The exosome fraction isolated by differential and buoyant density gradient centrifugation was assessed by transmission electron microscopy as described $[18,19]$. Exosome pellets were fixed in $3 \%(\mathrm{w} / \mathrm{v})$ glutaraldehyde and $2 \%$ paraformaldehyde in cacodylate buffer, $\mathrm{pH} 7.3$ and the sample was then applied to a continuous carbon grid and negatively stained with $2 \%$ uranyl acetate. The samples were examined in an FEI Tecnai 12 transmission electron microscope (FEI ${ }^{\mathrm{rm}}$, Hillsboro, Oregon, USA).

\section{Nanoparticle tracking analysis}

The size distribution of exosome preparations was analysed using a NanoSight LM10 system (NanoSight, Amesbury, UK) according to the manufacturer's instructions. Exosome samples isolated from OVCAR-3 and SKOV-3 cells were diluted in PBS $1 \mathrm{X}$ 1:3 and 1:6, respectively before the analysis. The instrument measured the rate of Brownian motion of nanoparticles [20].

\section{Quantification of ovarian cancer cell-derived exosome}

Exosome concentration was determined using the total exosomal CD63 protein measured by ELISA (ExoELISA ${ }^{\mathrm{TM}}$, System Biosciences, Mountain View, CA) according to the manufacturer's instructions. Quantitative results (number of exosome particles) were obtained using a exosome protein standard curve calibrated by NanoSight instrument provide by ExoELISA ${ }^{\mathrm{Tm}}$ kit.

\section{miRNA isolation}

Ambion mirVana PARIS Kit (Invitrogen, USA) was used to extract cellular and exosomal total RNA from OVCAR-3 and SKOV-3 by following the manufacturer's procedure. Cell samples were prepared by trypsinising the cells and centrifuging at $300 \times \mathrm{g}$ for $5 \mathrm{~min}$ to remove the supernatant. Samples are first lysed by adding cell disruption buffer and vortexed or pipetted vigorously. Denaturing solution was added to samples and incubated on ice for $5 \mathrm{~min}$. The first two steps stabilize RNA and inactivate RNases. The lysate is then subjected to Acid-Phenol: Chloroform extraction by adding Acid-Phenol:Chloroform, vortexed and centrifuged at $10,000 \times \mathrm{g}$ for $5 \mathrm{~min}$. Recovery of the aqueous phase obtains semi-pure RNA samples, removing most of the other cellular components. $100 \%$ ethanol was mixed and passed through a filter cartilage. The filter was washed three times and the RNA was eluted with nuclease-free water.

\section{Real-time PCR}

Reverse transcription was performed using the miScript Reverse Transcription Kit (QIAGEN, Valencia, CA, USA) in a total volume of $20 \mu \mathrm{l}$. cDNA was synthesised from the maximum volume of OVCAR-3 and SKOV-3 exosomal RNA $(12 \mu \mathrm{l})$ using the BIO-RAD T100 ${ }^{\text {TM }}$ Thermal Cycler (USA) running for $60 \mathrm{~min}$ at $37^{\circ} \mathrm{C}, 5 \mathrm{~min}$ for $95^{\circ} \mathrm{C}$ and $60 \mathrm{~min}$ for $37^{\circ} \mathrm{C}$. As the control, RNase-free water was added as the RNA template. Real-time PCR was performed with miScript SYBR Green Kit (QIAGEN, Valencia, CA, USA). Forward primers (miScript primer assays, QIAGEN, Valencia, CA, USA) designed to detect the following mature miRNAs were used: let-7 family (Hs_let-7a_2, Hs_let-7b_1, Hs_let-7c-1, Hs_let-7d_2, Hs_let-7e_3, and Hs_let-7f_1) and miR-200 family (Hs_miR-200a_1, Hs_ miR-200b_3, Hs_miR-200c_1). The universal primer included in the kit was used as the reverse primer. The reactions were performed in triplicate using the BIO-RAD $\mathrm{iQ}^{\mathrm{T}} 5$ Multicolor Real-Time PCR Detection System (USA) with the following conditions: $94^{\circ} \mathrm{C}$ for $3 \mathrm{~min}, 35$ amplification cycles of $94^{\circ} \mathrm{C}$ for $45 \mathrm{~s}, 55^{\circ} \mathrm{C}$ for $30 \mathrm{~s}$ and $72^{\circ} \mathrm{C}$ for $30 \mathrm{~s}$, $72^{\circ} \mathrm{C}$ for $10 \mathrm{~min}, 12^{\circ} \mathrm{C}$ for $\infty \mathrm{min}$. Let- $7 \mathrm{miRNA}$ and miR200 expression was normalized $\left(\Delta \mathrm{C}_{\mathrm{T}}\right)$ to the recommended 
housekeeping gene, human RNU6-2 (RNU6B) where $\Delta \mathrm{C}_{\mathrm{T}}=\mathrm{C}_{\mathrm{T} L E T-7 \text { or miR-200 }}-\mathrm{C}_{\mathrm{TRNU6B}}$. The data are presented as $2^{-\Delta C T}$. miRNA transcript expression from the same amount of RNA (Ambion mirVana PARIS Kit) was measured. RNU6-2 expression was consistent in all the samples both within and across experimental conditions. No statistically significant differences $(\mathrm{p}>0.05)$ in the expression of RNU6-2 between exosomes and/or cells samples measured by Standard Deviation of $C_{\mathrm{T}}$ were identified.

\section{Statistical analysis}

All studies conducted were treatment - control comparisons, with at least 3 independent experiments ( $n$ value) performed. Data are presented as mean \pm SEM ( $n$ value). Comparisons between two or more groups were performed by means of unpaired Student's $t$-test and analysis of variance (ANOVA), respectively. Statistical significance was defined at least $p<0.05$ determined using the GraphPad Prism software, Inc.

\section{Results}

\section{Characterisation of human epithelial ovarian cancer} cell lines

Initially, studies were conducted to confirm the invasiveness of the two ovarian cancer cell lines (OVCAR-3 and SKOV-3 cells) used in these studies. Figure 1A presents photomicrographs of wound closure over $24 \mathrm{~h}$ incubation for both cell lines and the percent change in relative wound density over time (Figure $1 \mathrm{~B})$. Figure $1 \mathrm{C}$ presents cell invasion expressed as area under the curve (AUC) of
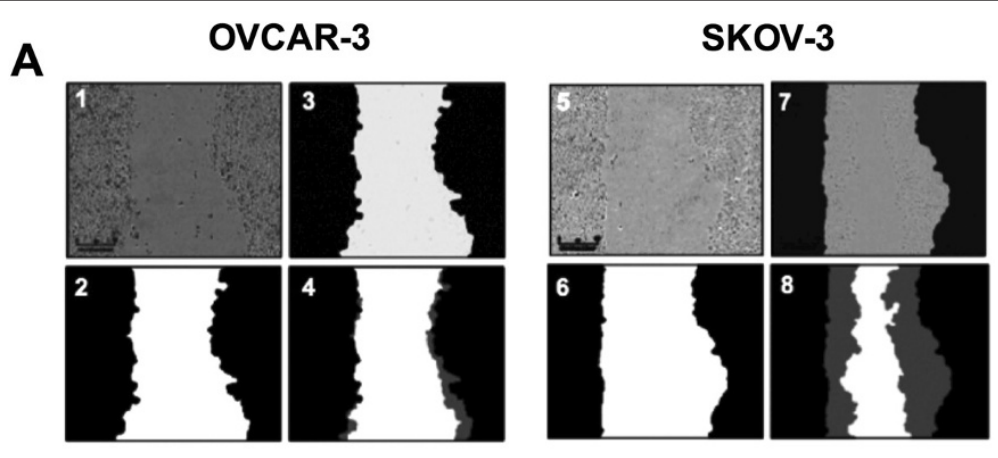

B

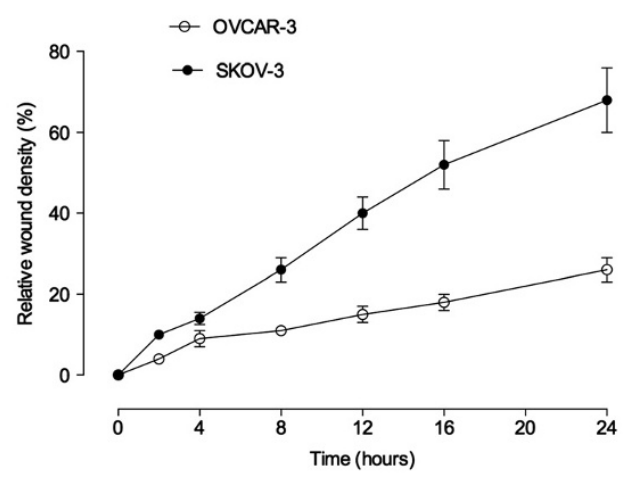

C

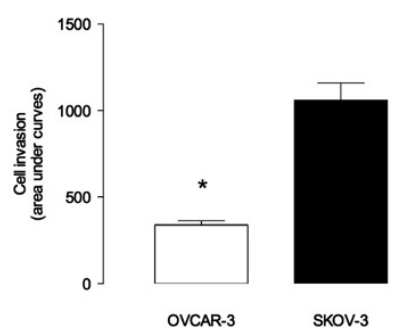

Figure 1 Ovarian cancer cell invasion. OVCAR-3 and SKOV-3 cells were grown to confluence in complete media. A wound was made using 96 well WoundMaker and then overlaid to form a 3D matrix-gel. Invasion assay was preformed with OVCAR-3 and SKOV-3 cells in complete media. Cells were imaged with IncuCyte ${ }^{T M}$ (Essen BioSciences, USA) every three hours for 24 h. (see Methods). (A) Representative images of OVCAR-3 (A1-A4) and SKOV-3 (A5-A8) cell invasion. A1 and A5: phase contrast micrograph image immediately after wounding; A2 and A6: graphical representation showing the calculation of initial wound width; A3 and A7: cells images show 24 hours later; A4 and A8: graphical representation of cell invasion at 24 hours of the experiment. The grey region denotes the area of the initial wound covered by advancing cells. (B) The time course of OVCAR-3 and SKOV-3 invasion. (C) Area under curves analysis from B. Data represented as mean \pm SEM $(n=6)$. In $\mathbf{C}$, ${ }^{*} P<0.001$ versus SKOV-3. 
the change in relative wound density overtime. AUC for SKOV-3 cell was 3.2 fold greater than the AUC for OVCAR-3 cells $(\mathrm{p}<0.0001)$. The data obtained are consistent with SKOV-3 cells being significantly more invasive than OVCAR-3 cells.

\section{Characterisation of ovarian cancer cell line-derived exosomes}

Exosomes derived from OVCAR-3 and SKOV-3 were isolated from cell-conditioned cultured media by differential and buoyant density centrifugation. The presence of exosomal markers, CD63 and CD9 in exosomes isolated from both cell lines was confirmed by Western blot (Figure 2A). The exosomal particulate fraction isolated from OVCAR-3 and SKOV-3 were examined under transmission electron microscopy. Exosomes were identified as small vesicles between $40-100 \mathrm{~nm}$ in a cup-shaped form from both cell lines (Figure 2B). Finally, we compared the size distribution of exosome preparations from OVAR-3 and SKOV-3 cells supernatants using a nanoparticle tracking analysis (NanoSight). OVAR-3 and SKOV-3 exosomes were characterised by a particle size range of $50-130 \mathrm{~nm}$ (Figure 2C).

\section{Exosome release from OVCAR-3 and SKOV-3 cells}

Exosomal protein release was expressed as total exosomal protein (i.e. particulate material with a buoyant density of 1.15 to $1.19 \mathrm{~g} / \mathrm{ml}$ ). OVCAR-3 cells released $0.44 \pm 0.05 \mu \mathrm{g}$ of exosomal protein per million cells per $24 \mathrm{~h}\left(n=6\right.$; i.e. 6 different isolations from $\sim 300 \times 10^{6}$ cells each) whereas SKOV-3 cells released $1.22 \pm 0.11 \mu \mathrm{g}$ of exosomal protein per million cells per $24 \mathrm{~h}(n=6$; i.e. 6 different isolations from $\sim 300 \times 10^{6}$ cells each) (Figure 3A). SKOV-3 cells released significantly more exosomes $(\sim 2.7$-folds $)$ in $24 \mathrm{~h}$ compared to OVCAR-3 cells $(\mathrm{p}<0.001)$. These results were confirmed using ELISA (see Methods). The number of exosome particles (NEP) released from SKOV-3 cells was $\sim 2.2$ fold higher than the release from OVACR-3 cells $\left(8.07 \times 10^{8} \pm 5.9 \times\right.$ $10^{7}$ vs $1.63 \times 10^{9} \pm 3.04 \times 10^{8}$ NEP in OVCAR-3 and

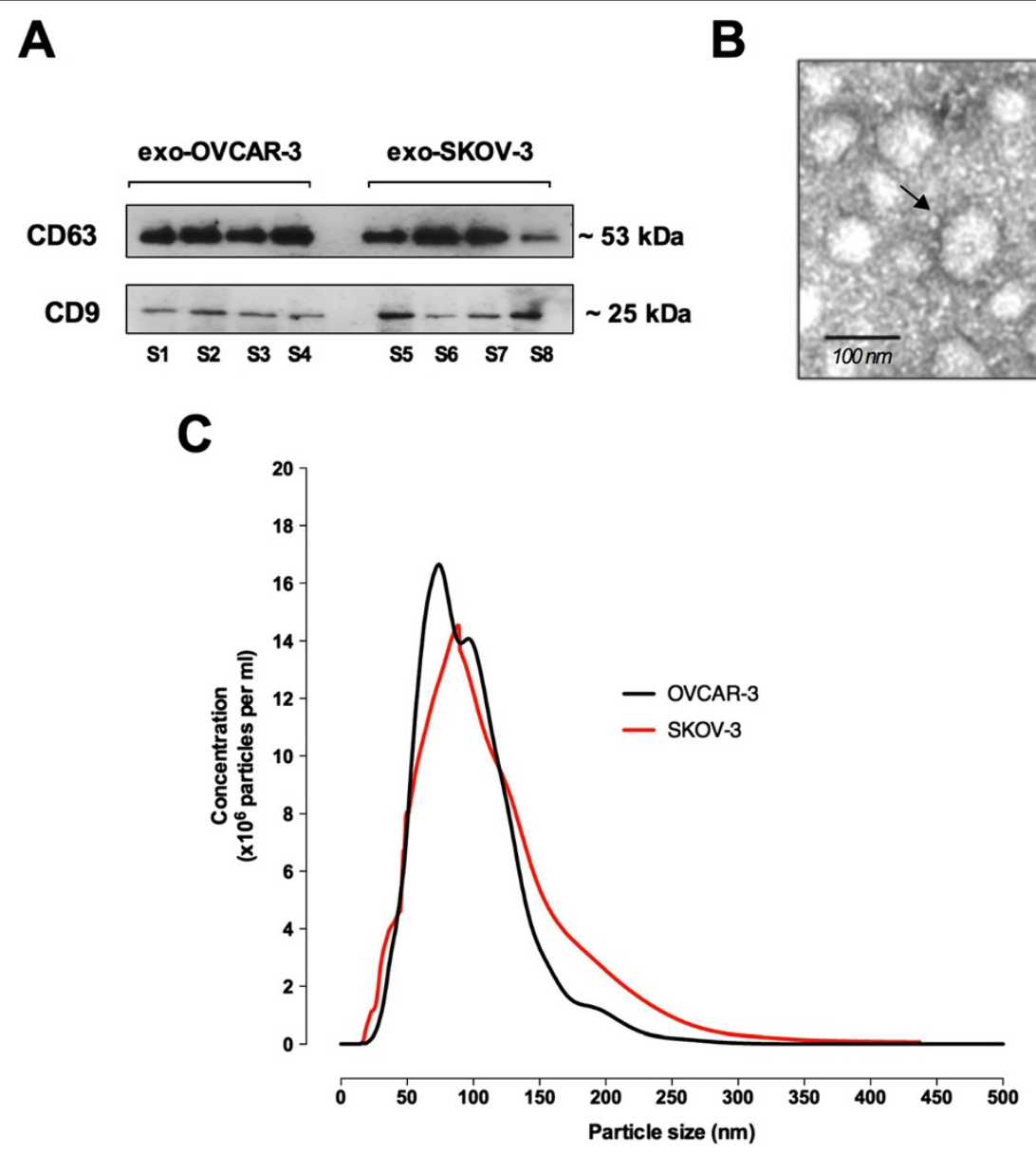

Figure 2 Characterisation of exosomes from ovarian cancer cell lines. (A) Representative image of western blot for the presence of CD63 and CD9. S1-S4 and S5-S6 represent different exosomes isolation from OVCAR-3 and SKOV-3 cells, respectively. (B) Representative electron micrograph of exosomes isolated from OVCAR-3 and SKOV-3 cells. (C) Nanosight measurement of particle-size distribution in preparation from OVAR-3 (black) and SKOV-3 (red) exosomes (see Methods). In B, Scale bar 100 nm. 
A

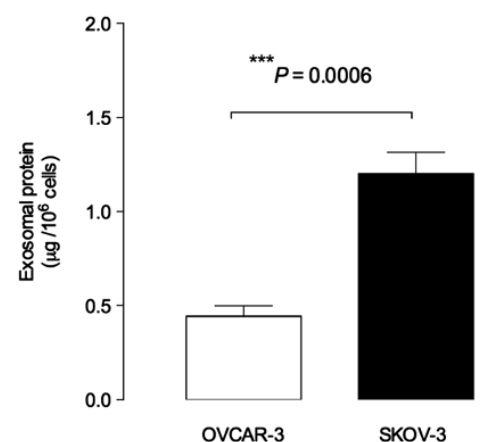

C
B

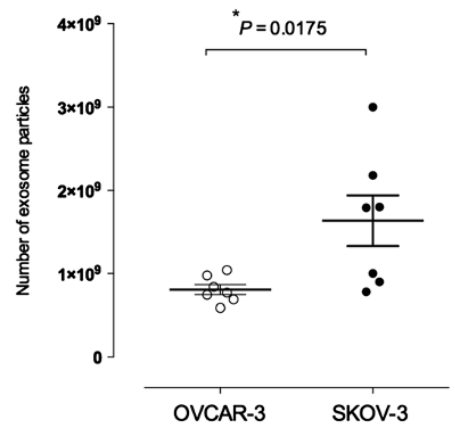

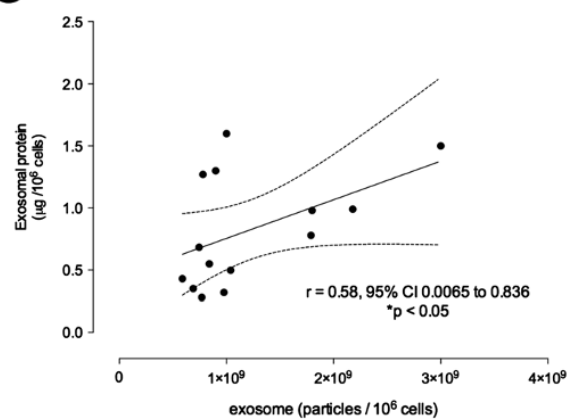

Figure 3 Exosome releases from ovarian cancer cell lines. Exosomes were quantified in culture media of ovarian cancer cell lines using an ELISA kit (see Methods). (A) Levels of exosome as presented as protein concentration from $1 \times 10^{6}$ OVCAR-3 (white bar) and SKOV-3 cells (black bar). (B) Quantification of number of exosome particles from OVCAR-3 (white circles) and SKOV-3 cells (black circles) per $10^{6}$ cells. (C) Relationship between number of exosome particles and exosomal protein concentration from OVCAR-3 and SKOV-3 cells. Fitted linear regression line with $95 \%$ confidence intervals (dotted lines). In $A,{ }^{* *} P<0.01$ versus SKOV-3. In $B,{ }^{*} P<0.05$ versus SKOV-3.

SKOV-3, respectively, $\mathrm{p}<0.05$ ) (Figure 3B). In addition, $\mathrm{NEP}$ and exosomal protein pellet concentration were significant correlated (Spearman's $\mathrm{r}=0.58 ; \mathrm{p}=0.037$ ).

\section{miRNA profile}

The presence and abundance of specific microRNA (let-7 and miR-200 family) in both OVCAR-3 and SKOV-3 cell-derived and exosome-derived were determined. The expression profile of miRNA (miR-let7 and miR-200) in the two cell lines was significantly different (ANOVA, $\mathrm{p}<0.001$ ). Post-hoc pairwise comparisons identified significant differences in the expression of miR-200 but not let-7 transcripts. (Bonferroni's Multiple comparison test, $\mathrm{p}<0.01$, Figure 4A).

In exosomes, miRNA expression profile (miR-let7 and miR-200) was significantly different between OVCAR3- and SKOV-3-derived exosomes. exo-SKOV-3 expressed significantly more $(n=6, p<0.001)$ let-7 transcripts compared to exo-OVCAR-3. Moreover, miR-200b and miR-200c were only expressed in exo-OVCAR-3 reflecting the miRNA signature in cells (Figure 4B). In addition, the expression of miRNA transcript was significantly higher in exosomes compared with the expression in cells (Figure 5A and $5 \mathrm{~B})$.

\section{Discussion}

The aim of this study was to test the hypothesis that ovarian cancer cell invasiveness is associated with altered release of exosomes and discordant exosomal sequestration of miRNA. Two ovarian cancer cell lines of epithelial cell origin but with different invasive potentials were used to test this hypothesis. In this study, the release of exosomes from cells incubated under similar conditions, was $\sim 2$-folds greater from SKOV-3 cells (high invasive) than from OVCAR-3 cells (low invasive). Exosomes released by SKOV-3 cells contained a greater abundance of let-7 miRNA transcripts than OVCAR-3 cells. When exosomal miRNA content was compared with that of the cell of origin, SKOV-3 cell-derived exosomes displayed no correlation with the cell origin. In contrast, the miRNA profiles of OVCAR-3 cell-derived exosomes and their cells of origin were highly correlated. The data obtained established that the release and miRNA content of exosomes differs significantly between ovarian cancer cell lines and correlates with their invasive potential. Furthermore, the data are consistent with the hypothesis that selective packaging of specific miRNA transcripts into exosomes may represent a regulatory mechanism to maintain invasive phenotype by reducing the intracellular availability 


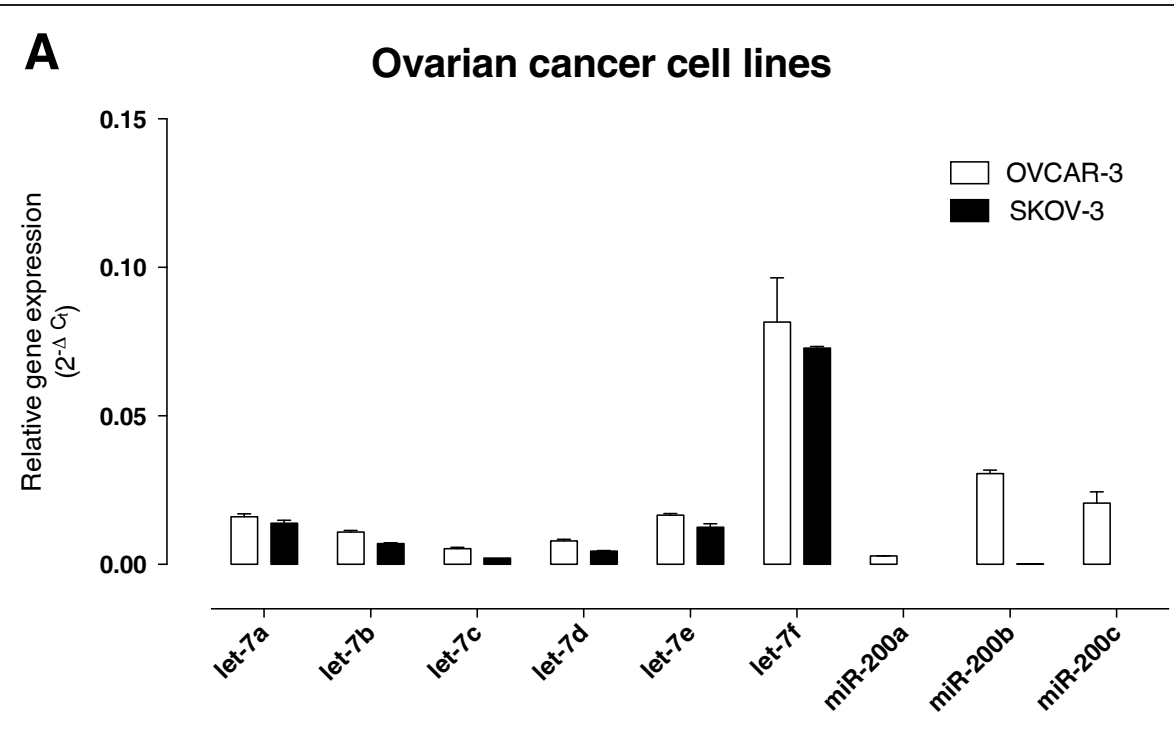

B

exosomes

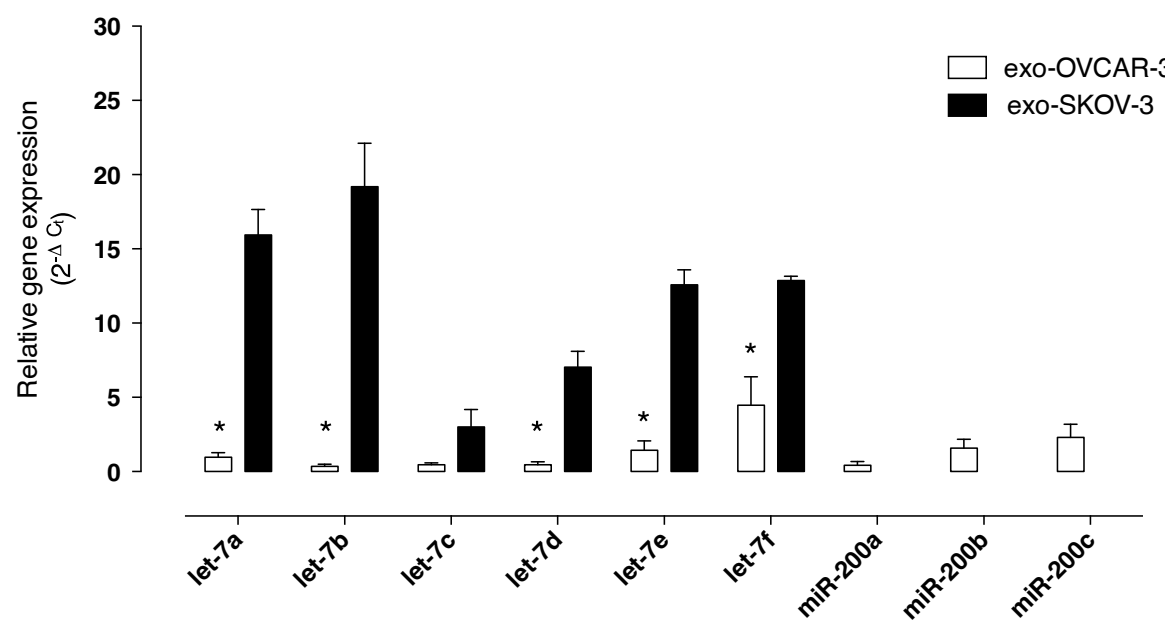

Figure 4 Expression of let-7 and miR-200 families. Total microRNA was extracted using the Ambion mirVana PARIS Kit (Invitrogen). Real-time PCR was performed with miScript SYBR Green Kit to compare let-7 and miR-200 family expression between ovarian cancer cell lines (OVAR-3 and SKOV-3 cells) (A) and between cell-derived exosomes (exo-OVAR-3 and exo-SKOV-3) (B). Data are represented as mean \pm SEM $(n=6)$. In $\mathbf{A}$ and $\mathbf{B}$, ANOVA $p<0.0001$ between groups. In $\mathbf{B},{ }^{*} p<0.001$ vs exo-SKOV-3.

of miRNAs (i.e. let-7 miRNAs) with known tumor suppressor activity.

In this study, we initially confirmed that SKOV-3 have a greater capacity to invade than OVCAR-3 cells. Using a $3 \mathrm{D}$ in vitro culture model, we observed that SKOV-3 cells were 2-3 times more invasive than OVCAR-3 cells ( $\mathrm{p}<$ $0.0001)$. These data are consistent with previous publications [17].

Exosomes released from ovarian cancer cells in vitro were isolated and characterised using well-established methods [9,18,19,21-23]. Exosomes isolated from both cell lines displayed characteristic morphology, buoyant density (1.15 to $1.19 \mathrm{~g} / \mathrm{ml}$ ) and exosomal markers (i.e.
CD63 and CD9 - members of the tetraspanin family and one of the most abundant protein families present in exosomes [24]. Previously, we [19] and others [7,21,25] have successfully used such preparation of exosomes for both proteomic analysis and RNA profiling.

In this study, we defined the release of exosomes from ovarian cancer cells as the accumulation (in exosome-free incubation medium) of CD63 and CD9 positive, particulate protein with a density of 1.15 to $1.19 \mathrm{~g} / \mathrm{ml}$. Exosome release was cell type specific and correlated with cell invasiveness. When cells were incubated under identical conditions, the release of exosomes from SKOV-3 cells was more than 2-folds greater than that of OVCAR-3 cells 


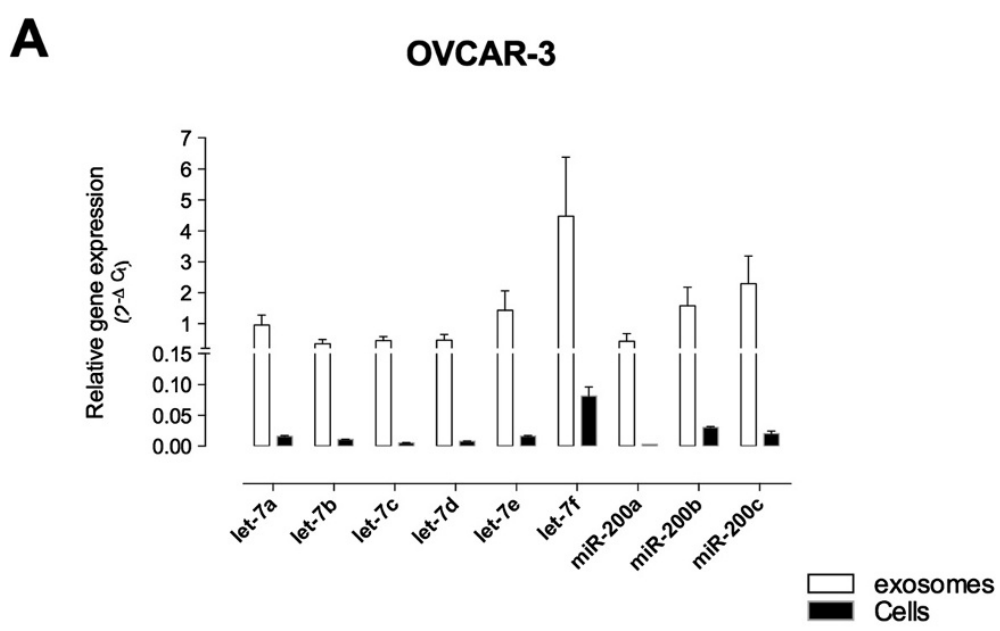

B

SKOV-3

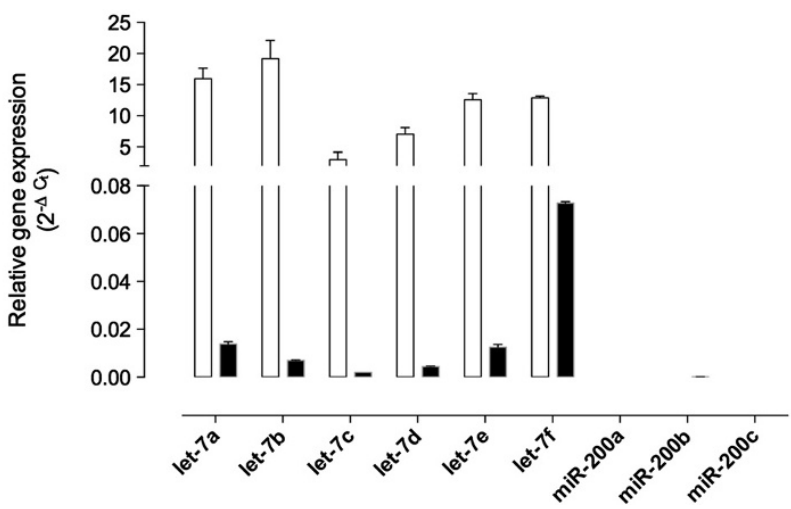

Figure 5 Comparison of miRNA transcripts expression between cells and exosomes. The miRNA expressions between cells and exosomes in OVCAR-3 (A) and SKOV-3 (B). Data is represented by mean \pm SEM ( $n=6$ per group).

$(\mathrm{p}<0.001)$. Exosome have been previously isolated from both OVCAR-3 [7,26] and SKOV-3 cells [9], however, this is the first study to directly compare exosome release from these cell lines.

In vivo, exosomes released from ovarian tumours may be present in blood and other biological fluids. Previously, Taylor and Gercel-Taylor [9] isolated exosomes from serum samples of women with ovarian cancer. The concentration of exosomes was significantly elevated with disease progression. The release of exosomes has also been found to correlate with the invasiveness of other tumour cell lines [27]. Even though the molecular mechanism triggering the release of exosomes is poorly understood, factors promoting exosome release have been identified, including: hypoxia, anticancer drugs, thermal and oxidative stress [28-31]. It is well established that heterogeneity exists among tumour cells even in a single tumour [16].

This study further characterised ovarian cancer cell exosome content by RT-PCR (miRNA content). Of particular note was the variation in expressions of two miRNA families the let-7 and miR-200 transcripts. Iori, Visone et al. [16] performed a large scale miRNA profiling of ovarian cancer tissues. Let-7 was found to be down-regulated and miR-200 transcripts were among the most significantly up-regulated in epithelial ovarian cancer tissues compared to normal tissues [16]. Both let-7 and miR-200 families are tumour suppressors; let-7 repressing cell proliferation while miR-200 regulate epithelial to mesenchymal transition [32]. Taylor and Gercel-Taylor [9] has compared the expression of selected miRNA in exosomes and originating cells. Expression levels of most miRNA including let-7a, b, c, d, f and miR-200a, b, c have been found to be the same, whereas let-7e was elevated in exosomes compared to the cell, suggesting active selection during miRNA compartmentalisation into exosomes [28]. The overall expressions of let-7 transcripts were more abundant in OVCAR-3 cells than SKOV-3 cells. Interestingly, in exosomes, let-7 transcripts were more abundant in SKOV-3 cell derived exosomes. The low expression of let-7 in SKOV-3 cells may be due to the compartmentalisation into exosomes. Exosomes can either fuse with the lysosomes to degrade their contents or release them out 
of the cells to decrease the expression of tumour suppressor miRNAs, ultimately leading to their aggressive phenotype. The miR-200 transcripts were only present in exosomes from OVCAR-3 as SKOV-3 did not express miR-200 s in their cells. Previous studies have compared miRNA expression in normal and cancerous tissue or cell lines but have not compared expressions of different metastatic potentials.

Recently, we demonstrated that proteins are selectively packaged into placental cells exosomes by a process that, at least in part, is regulated by the extracellular milieu (i.e. low oxygen tension) [18,33]. In addition, Li et al., (2013) [34] reported that microvesicles (i.e. exosomes) from glioma cells contain selective packaged miRNA that modify the gene expression in cell target [34]. These observations are consistent with data obtained in this study and the hypothesis that exosomal oligonucleotides are selective packaged into exosome by endosomal process (that are yet to be identified).

In this study, the expression of let-7 miRNA transcripts was similar in both parent cell lines, however, miR-200 transcripts were only identified in OVCAR-3 cells. In contrast, exosomal expression of both let- 7 and 200 miRNA transcripts displayed a dramatic cell specific variation. The expression of let-7 miRNA transcripts in exosomes released from SKOV-3 cells was 7-20 times greater than in exosomes released from OVCAR-3 cells. Similar, to the expression profile in the parent cells, miR-200 transcripts were only identified in exosomes from OVCAR-3. These data are suggestive that ovarian cancer cell invasiveness is not affected by the expression of let-7 miRNA transcripts but that repression of miR-200 transcripts is associated with increased invasive capacity. Furthermore, the data obtained establish the selective and cell specific packaging of miRNA transcripts into exosomes.

The expression of miRNA-200 transcripts in OVCAR-3 cells and their absence in the more aggressive SKOV-3 cell line is consistent with the proposed involvement of miR$200 \mathrm{~s}$ in tumor suppression via the inhibition of epithelialmesenchymal transition (EMT), the initiating step of metastasis [32]. The expression of miR-200 s in OVCAR-3 may be a contributing factor in determining their lower invasive potential when compared to SKOV-3 cells. In support of this suggestion, the let-7 miRNA transcripts a-f were identified in both cell types but were more abundant in OVCAR-3.

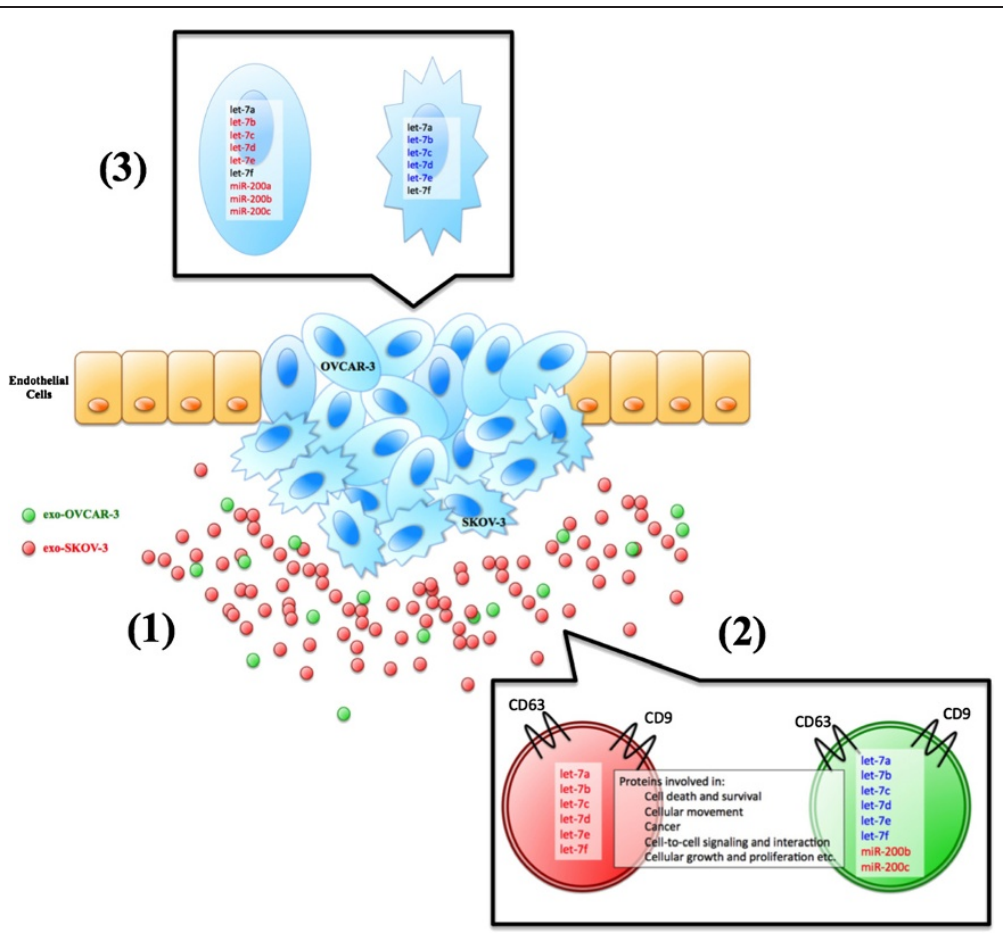

Figure 6 Proposed model of ovarian cancer cell-derived exosome action. Tumour cells with different invasion capacity exist in a single epithelial ovarian tumour, releasing exosomes. High invasive tumour cells (SKOV-3) release significantly more exosomes (1), containing proteins involved in processes such as: cell death and survival, cellular movement, cancer, cell-to-cell signalling and interaction, cellular growth and proliferation. The let-7 family that are known to suppress cell proliferation was significantly more expressed in exosomes from high invasive exosomes (from SKOV-3). On the other hand, the miR-200 family that suppress epithelial to mesenchymal transition was only expressed in exosomes derived from low invasive cells (from OVCAR-3) (2). The miRNA profile differs between exosomes derived from cells with different invasive capacity (OVAR-3 versus SKOV-3) (3). 


\section{Conclusions}

Based on the data obtained in this study and on previously published observations, we propose the following model of ovarian cancer cell exosome release and action (Figure 6). Tumour cells with different invasion capacity exist in a single epithelial ovarian tumour, releasing exosomes. High invasive tumour cells (SKOV-3) release significantly more exosomes [1], containing proteins involved in processes such as: cell death and survival, cellular movement, cancer, cell-to-cell signalling and interaction, cellular growth and proliferation. The let-7 family that is known to suppress cell proliferation was significantly more expressed in exosomes from high invasive exosomes (from SKOV-3). On the other hand, the miR-200 family that suppresses epithelial to mesenchymal transition was only expressed in exosomes derived from low invasive cells (from OVCAR3) [2]. The miRNA profile differs between exosomes derived from cells with different invasive capacity (OVCAR-3 versus SKOV-3) (3). Interestingly, significantly less let-7 family miRNA was expressed in the high invasive cells (SKOV-3) although it was highly expressed in exosomes. Within the tumour, high invasive tumour cell derivedexosomes signal low invasive tumour cells and low invasive tumour cell derived-exosomes can signal high invasive tumour cells to increase invasion of recipient cells.

\section{Competing interests}

The authors declare that they have no competing interests.

\section{Author's contributions}

MK and CS contributed in generating experimental data. MK, CS, JT, SEI, MDM, and GER contributed in discussion and reviewed/edited manuscript. MK, CS and GER wrote the manuscript and drew the figures. All authors read and approved the final manuscript.

\section{Acknowledgements}

This work was partially funded by grants from: the Department of Industry, Innovation, Climate Change, Science, Research and Tertiary Education, Australian Government; Smart Futures, Queensland Government; and RoCan (http://rocan.com.au). The authors grateful acknowledge the long-standing support of the members Rotary Club of Williamsontown, Victoria, Australia. Dr Salomon is in receipt of a University of Queensland Post-doctoral Fellowship (2013-2015). Prof Rice is in receipt of an NHMRC Principal Research Fellowship (2010-2014).

\section{Author details}

${ }^{1}$ Centre for Clinical Diagnostics, Royal Brisbane and Women's Hospital, University of Queensland Centre for Clinical Research, Building 71/918, Herston, Queensland 4029, Australia. ²Department of Obstetrics and Gynaecology, Faculty of Medicine, Universidad de los Andes, Santiago, Chile.

Received: 18 July 2013 Accepted: 18 December 2013 Published: 6 January 2014

\section{References}

1. Jemal A, Bray F, Center MM, Ferlay J, Ward E, Forman D: Global cancer statistics. CA Cancer J Clin 2011, 61:69-90.

2. Siegel R, Naishadham D, Jemal A: Cancer statistics, 2012. CA Cancer J Clin 2012, 62:10-29.

3. Jemal A, Siegel R, Ward E, Hao Y, Xu J, Thun MJ: Cancer statistics, 2009. CA Cancer J Clin 2009, 59:225-249.

4. Taylor DD, Gercel-Taylor C: Tumour-derived exosomes and their role in cancer-associated T-cell signalling defects. Br J Cancer 2005, 92:305-311.
5. Simpson RJ, Jensen SS, Lim JW: Proteomic profiling of exosomes: current perspectives. Proteomics 2008, 8:4083-4099.

6. Lässer C, Seyed Alikhani V, Ekström K, Eldh M, Torregrosa Paredes P, Bossios A, Sjöstrand M, Gabrielsson S, Lötvall J, Valadi H: Human saliva, plasma and breast milk exosomes contain RNA: uptake by macrophages. J Transl Med 2011, 9:9.

7. Alvarez ML, Khosroheidari M, Kanchi Ravi R, DiStefano JK: Comparison of protein, microRNA, and mRNA yields using different methods of urinary exosome isolation for the discovery of kidney disease biomarkers. Kidney Int 2012, 82:1024-1032.

8. Thery C, Boussac M, Veron P, Ricciardi-Castagnoli P, Raposo G, Garin J, Amigorena S: Proteomic analysis of dendritic cell-derived exosomes: a secreted subcellular compartment distinct from apoptotic vesicles. J Immunol 2001, 166:7309-7318.

9. Taylor DD, Gercel-Taylor C: MicroRNA signatures of tumor-derived exosomes as diagnostic biomarkers of ovarian cancer. Gynecol Oncol 2008, 110:13-21.

10. Paranjape T, Slack FJ, Weidhaas JB: MicroRNAs: tools for cancer diagnostics. Gut 2009, 58:1546-1554.

11. Ambros $V$ : The functions of animal microRNAs. Nature 2004, 431:350-355.

12. Bartel DP: MicroRNAs: genomics, biogenesis, mechanism, and function. Cell 2004, 116:281-297.

13. Miska EA: How microRNAs control cell division, differentiation and death. Curr Opin Genet Dev 2005, 15:563-568.

14. Chan HW, Lappas M, Yee SW, Vaswani K, Mitchell MD, Rice GE: The expression of the let-7 miRNAs and Lin28 signalling pathway in human term gestational tissues. Placenta 2013, 34:443-448.

15. Helland A, Anglesio MS, George J, Cowin PA, Johnstone CN, House CM, Sheppard KE, Etemadmoghadam D, Melnyk N, Rustgi AK, et al: Deregulation of MYCN, LIN28B and LET7 in a molecular subtype of aggressive high-grade serous ovarian cancers. PLoS One 2011, 6:e18064.

16. Iorio MV, Visone R, di Leva G, Donati V, Petrocca F, Casalini P, Taccioli C, Volinia S, Liu CG, Alder H, et al: MicroRNA signatures in human ovarian cancer. Cancer Res 2007, 67:8699-8707.

17. Choi JH, Choi KC, Auersperg N, Leung PC: Gonadotropins upregulate the epidermal growth factor receptor through activation of mitogenactivated protein kinases and phosphatidyl-inositol-3-kinase in human ovarian surface epithelial cells. Endocr Relat Cancer 2005, 12:407-421.

18. Salomon C, Ryan J, Sobrevia L, Kobayashi M, Ashman K, Mitchell M, Rice GE: Exosomal signaling during Hypoxia mediates microvascular endothelial cell migration and vasculogenesis. PLoS One 2013, 8:e68451.

19. Thery C, Amigorena S, Raposo G, Clayton A: Isolation and characterization of exosomes from cell culture supernatants and biological fluids. Curr Protoc Cell Biol 2006, Chapter 3(Unit 3):22.

20. Soo CY, Song Y, Zheng Y, Campbell EC, Riches AC, Gunn-Moore F, Powis SJ: Nanoparticle tracking analysis monitors microvesicle and exosome secretion from immune cells. Immunology 2012, 136:192-197.

21. Atay S, Gercel-Taylor C, Kesimer M, Taylor DD: Morphologic and proteomic characterization of exosomes released by cultured extravillous trophoblast cells. Exp Cell Res 2011, 317:1192-1202.

22. Gercel-Taylor C, Atay S, Tullis RH, Kesimer M, Taylor DD: Nanoparticle analysis of circulating cell-derived vesicles in ovarian cancer patients. Anal Biochem 2012, 428:44-53

23. Thery C, Zitvogel L, Amigorena S: Exosomes: composition, biogenesis and function. Nat Rev Immunol 2002, 2:569-579.

24. Liang B, Peng P, Chen S, Li L, Zhang M, Cao D, Yang J, Li H, Gui T, Li X, Shen $\mathrm{K}$ : Characterization and proteomic analysis of ovarian cancer-derived exosomes. J Proteomics 2013, 80C:171-182.

25. Szajnik M, Derbis M, Lach M, Patalas P, Michalak M, Drzewiecka H, Szpurek D, Nowakowski A, Spaczynski M, Baranowski W, Whiteside TL: Exosomes in plasma of patients with ovarian carcinoma: potential biomarkers of tumor progression and response to therapy. Gynecol Obstet 2013, S4:003. doi:10.4172/2161-0932.S4-003.

26. Escrevente C, Keller S, Altevogt P, Costa J: Interaction and uptake of exosomes by ovarian cancer cells. BMC Cancer 2011, 11:108.

27. Ginestra A, Fau - La Placa MD, La Placa M, Fau - Saladino F, Saladino F, Fau - Cassara D, Cassara D, Fau - Nagase H, Nagase H, Fau - Vittorelli ML: The amount and proteolytic content of vesicles shed by human cancer cell lines correlates with their in vitro invasiveness. Anticancer Res 2011, 18:3433-3437

28. King HW, Michael MZ, Gleadle JM: Hypoxic enhancement of exosome release by breast cancer cells. BMC Cancer 2012, 12:421. 
29. Hedlund M, Nagaeva O, Kargl D, Baranov V, Mincheva-Nilsson L: Thermaland oxidative stress causes enhanced release of NKG2D ligand-bearing immunosuppressive exosomes in leukemia/lymphoma $T$ and $B$ cells. PLoS One 2011, 6:e16899.

30. Lv LH, Wan YL, Lin Y, Zhang W, Yang M, Li GL, Lin HM, Shang CZ, Chen YJ, Min J: Anticancer drugs cause release of exosomes with heat shock proteins from human hepatocellular carcinoma cells that elicit effective natural killer cell antitumor responses in vitro. J Biol Chem 2012, 287:15874-15885.

31. Fidler IJ, Kripke ML: Metastasis results from preexisting variant cells within a malignant-tumor. Science 1977, 197:893-895.

32. Zaman MS, Maher DM, Khan S, Jaggi M, Chauhan SC: Current status and implications of microRNAs in ovarian cancer diagnosis and therapy. J Ovarian Res 2012, 5(1):44.

33. Salomon C, Kobayashi M, Ashman K, Sobrevia L, Mitchell M, Rice G: Hypoxia-Induced Changes in the Bioactivity of Cytotrophoblast-derived Exosomes. PloS One 2013, 8(11):e79636.

34. Li CC, Eaton SA, Young PE, Lee M, Shuttleworth R, Humphreys DT, Grau GE, Combes V, Bebawy M, Gong J, et al: Glioma microvesicles carry selectively packaged coding and non-coding RNAs which alter gene expression in recipient cells. RNA Biol 2013, 10(8):1333-1344.

doi:10.1186/1479-5876-12-4

Cite this article as: Kobayashi et al:: Ovarian cancer cell invasiveness is associated with discordant exosomal sequestration of Let-7 miRNA and miR-200. Journal of Translational Medicine 2014 12:4.

\section{Submit your next manuscript to BioMed Central and take full advantage of:}

- Convenient online submission

- Thorough peer review

- No space constraints or color figure charges

- Immediate publication on acceptance

- Inclusion in PubMed, CAS, Scopus and Google Scholar

- Research which is freely available for redistribution 